\title{
Assessment of Elbow Joint Kinematics in Passive Motion by Electromagnetic Motion Tracking
}

\author{
$* \dagger$ M. Bottlang, $\ddagger$ S. M. Madey, §C. M. Steyers, §J. L. Marsh, and $† \S T$. D. Brown \\ *Biomechanics Laboratory, Legacy Clinical Research and Technology Center, Portland; Departments of \\ $\dagger$ Biomedical Engineering and \$Orthopaedic Surgery, University of Iowa, Iowa City, Lowa; and \\ tDepartment of Trauma Surgery, Legacy Emanuel Hospital, Portland, Oregon, U.S.A.
}

\begin{abstract}
Summary: This research provides a detailed analysis of the kinematics of passive elbow motion. Il quantifies how closely humeroulnar kinematics approximates rotation around a fixed axis. The results are clinically relevant for emerging treatment modalities that impose an artificial hinge to the elbow joint, such as total elbow arthroplasty and articulated external fixation. In a cadaveric study of seven specimens, we quantified uInar rotation around the humerus in terms of instantaneous screw displacement axes calculated from electromagnetic motion-tracking source data. This methodology enabled description of the complex excursion of the elbow axis in terms of translation and orientation changes of the screw displacement axes over the range of motion. Furthermore, we analyzed the envelope of joint laxity for elbow motion under applied small varus and valgus moments. In addition, radiographic landmarks of clinical utility for axis location were evaluated by visualizing the elbow's radiographic appearance when viewed from along the calculated best-fit (average) rotation axis. Over the normal range of elbow motion, the screw displacement axis varied 2.6-5.7 in orientation and 1.4-2.0 $\mathrm{mm}$ in translation. All instantaneous rotation axes nearly intersected on the medial facet of the trochlea. The breadth of the envelope of varus-valgus joint laxity was greatest within the initial $40^{\circ}$ of flexion and decreased by a factor of approximately two for flexion angles exceeding $100^{\circ}$.
\end{abstract}

A number of operative techniques have recently emerged that impose an artificial hinge on the elbow joint. Currently, these include articulated external fixation (e.g., the Compass Hinge; Smith and Nephew, Memphis, TN, U.S.A.) and distraction arthroplasty (14). The success of these emerging treatment modalities depends on precise alignment of the imposed artificial hinge with the natural elbow rotation axis, since the elbow is the articulation that most closely resembles a perfect hinge joint in the human. This study describes how a single fixed axis can be established that best approximates the complex kinematics of passive elbow motion. Furthermore, the deviation of the instant axis of joint rotation from such a single axis is quantitatively analyzed for various experimental parameters, such as direction of elbow motion (flexion or extension), motion under applied varus or valgus moments, and simulated muscle-load configurations (predominantly flexor or extensor forces). The determination of a single approximate elbow axis, in conjunction with the detailed documentation of passive elbow kinematics under consideration of

Reccived July 13, 1998; accepted July 28, 1999.

Address correspondence and reprint requests to T. D. Brown at Orthopaedic Biomechanics Laboratory, University of Iowa, 2181 E. Westlawn, Iowa City, IA 52242, U.S.A. E-mail: tom-brown@ uiowa.edu various constraint parameters, provides results relevant for the design and application of total elbow replacements, hinged external fixators, and distraction arthroplasty.

Previously, the axis of rotation of the elbow was estimated by London (11) in a two-dimensional analysis, using planar radiographs and the instant center technique. Morrey and Chao (13) assessed elbow joint motion with biplanar roentgenograms obtained at various flexion angles. They reported three-dimensional joint kinematics; however, imprecision in source data due to radiographic tracing of multiple finite-size markers limited the accuracy of their estimates. Deland et al. (8) determined the average axis of elbow rotation in three-dimensional space using time-lapse photographs of active luminescent marker triads. Despite having three-dimensional source data, however, they approximated the axis location by utilizing a modified instant center two-dimensional technique.

All these results quantitatively refined rather than conceptually extended the early findings of Fischer (9), who in 1911 stated that the instant center of elbow rotation remains within a locus less than $3 \mathrm{~mm}$ in diameter. He concluded that, for practical purposes, the elbow joint approximates a fixed hinge joint, with its axis located at the center of the trochlea.

More recently, alternating-current electromagnetic 


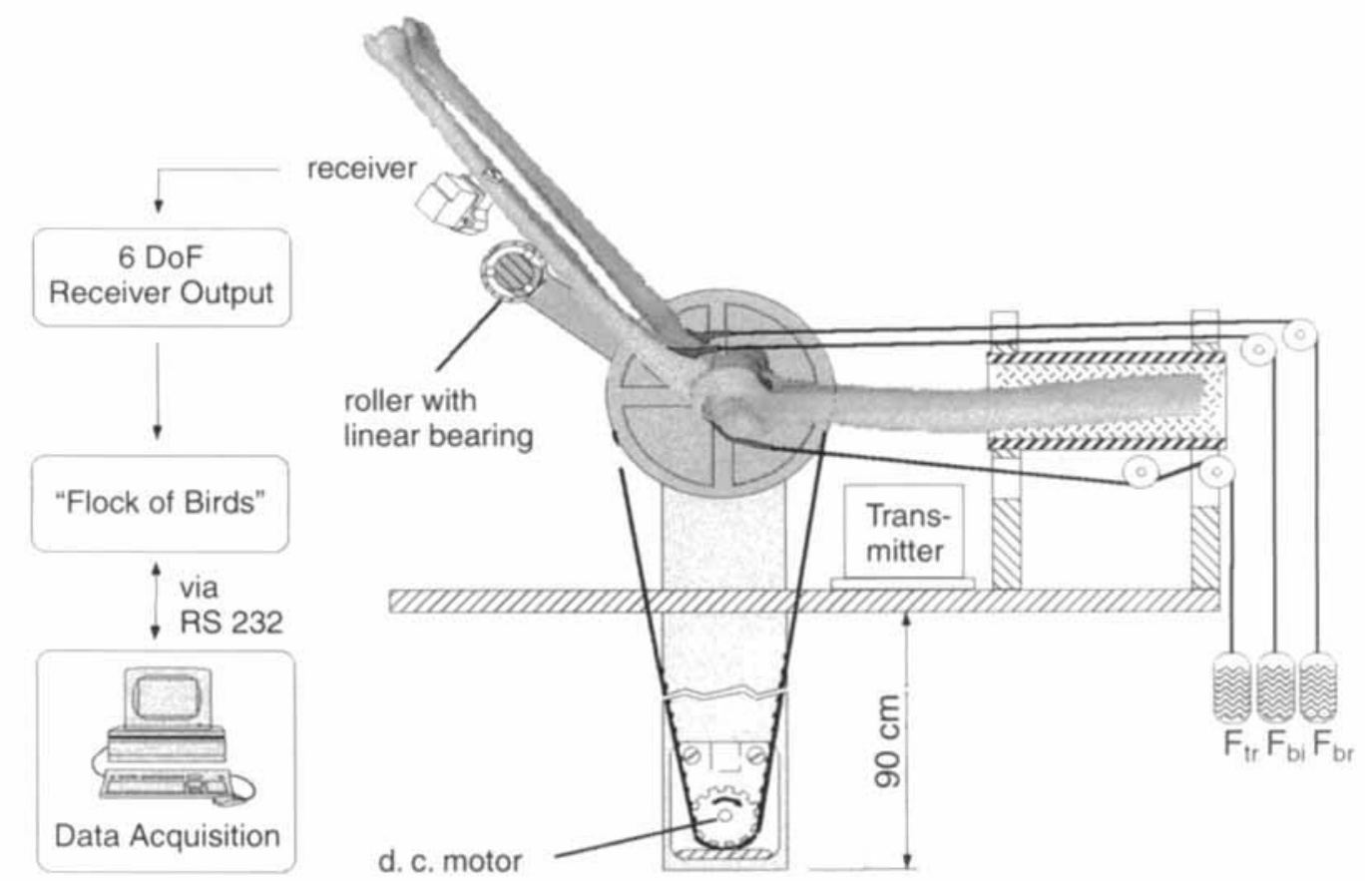

FIG. 1. Test setup for the application of minimally restrained passive elbow motion, shown for roller configuration $A$. DoF $=$ degrees of freedom, RS $232=$ connection type, $d . c$. $=$ direct current, $F_{t r}=$ triceps force, $F_{b i}=$ biceps force, and $F_{b r}=$ brachialis force. Flock of Birds electromagnetic tracking system (Ascension Technology).

motion tracking has been applied to the elbow joint $(2,10,16,18)$. However, most of these studies focused on comparing specific kinematic parameters before and after total elbow arthroplasties. Tanaka et al. (18) were the first to describe three-dimensional elbow kinematics, obtained from electromagnetic motiontracking data. Their data analysis, based on Eulerian angles, provided a thorough description of the orientation angles of the joint axis. However, even in the elbow, the joint axis translates as well as rotates, and translation motions are not accessible in an Eulerianangle approach.

In this study, we introduce the application of directcurrent electromagnetic motion tracking to continuously trace dynamic, passive motion of the natural. elbow joint. The high spatial resolution of this system, in combination with a customized method for processing the data after the procedure, allows a comprehensive description of the pathway of the instant axis of elbow rotation. Besides providing a computational

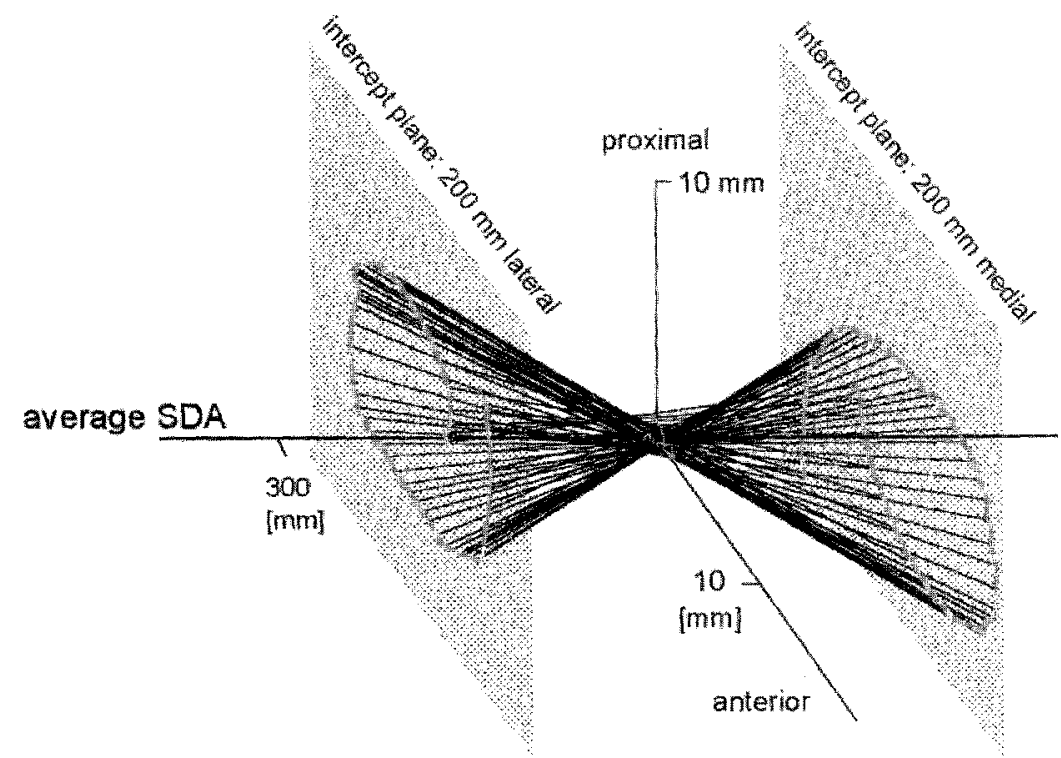

FIG. 2. Pathway of the screw displacement axis (SDA) with respect to the humerus during clbow flexion from $10 \mathrm{lo} 130^{\circ}$ in roller configuration A. For visualization, the excursion of the axis is exaggcrated by tracing the projection onto planes located $200 \mathrm{~mm}$ medial and lateral of the trochlea. Note the large scaling difference within, compared with perpendicular to, these interccpt planes. 


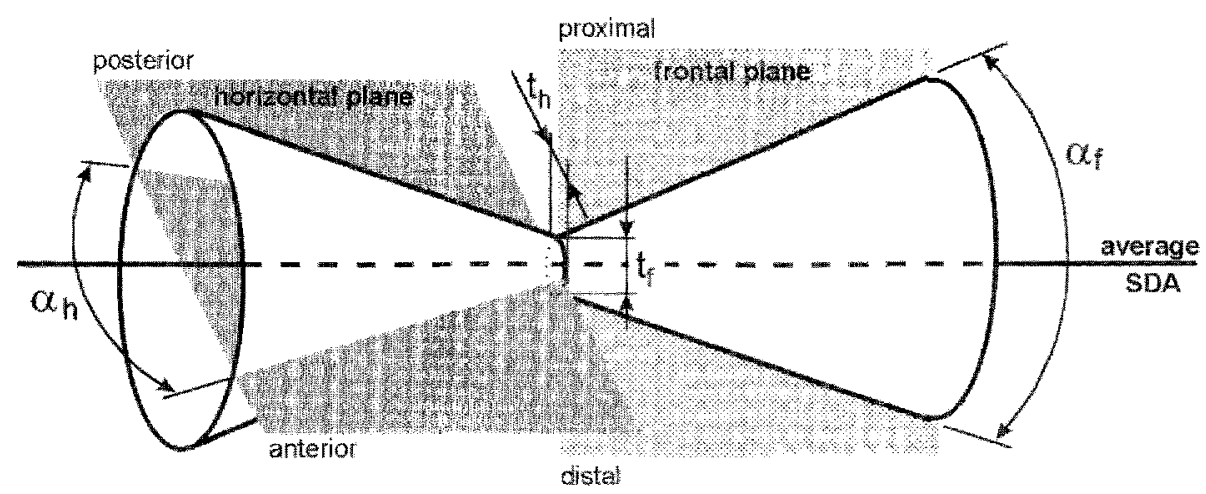

FIG. 3. Idealization of the pathway of the screw displacement axis (SDA) in terms of a quasi-conic double frustum to establish distinctive excursion parameters for translation and changes in oricntation. $\alpha_{h}$ and $\alpha_{f}=$ frustum vertex angles, and $t_{h}$ and $t_{f}=$ translations of the screw displacement axis in the horizontal and frontal planes, respectively.

approach to extract an average rotations axis from the instant axes of joint rotation, we also demonstrate the radiographic appearance of the elbow when viewed from along such a calculated average rotation axis.

\section{MATERIALS AND METHODS}

Seven fresh-frozen cadaveric upper extremities with no radiographic or visual evidence of pathologic conditions werc amputated at the mid-humeral shaft and disarticulated at the radiocarpal joint. The skin, subcutaneous tissues, and muscles were excised, and the joint capsule, ligaments, and musculotendinous insertions of the biceps, brachialis, and triceps were retained. The mid-diaphysis of the humcrus was secured with polymethylmethacrylate in a Plexiglas tube, which in turn was rigidly affixed to a specially designed elbow-motion applicator (Fig. 1). This experimental setup, driven by a direct-current motor, allowed minimally constrained rotation, without manual interference, through the elbow's range of motion. Elbow rotation was applicd at a constant angular velocity $(32 \% \mathrm{sec})$ to control for the viscoelastic (i.e., rate-dependent) material properties of the tissues constraining the joint. Consistency of angular velocity proved essential to establish reproducible recordings: of the envelope of joint laxity.

A mobile roller, contacting the forearm $150 \mathrm{~mm}$ distal to the clbow joint and rotating around an axis that was approximately aligned with the natural elbow axis, induced passive flexion or extension of the elbow. A linear glass-ball bearing atop the roller cnabled unrestricted varus/valgus motion of the forearm. Weights were attached to the musculotendinous insertions by nylon cables to simulate a constant applied biceps force $\left(\mathrm{F}_{\mathrm{b} i}\right)$, brachialis force $\left(F_{h r}\right)$, and triceps force $\left(F_{t r}\right)$. These muscle forces (1) were simulated to ensure continuous contact at the articular surface, as well as contact between the forearm and the roller. The nylon cables were guided in a manner so as to approximate normal muscle moment arms (15).

Elbow rotation was achieved in two distinct roller configurations: A and B. In configuration A, a roller located posteriorly with respect to the ulna guided the forearm into flexion and extension while a predominant extensor corce $\left(\mathrm{F}_{\mathrm{bi}}+\mathrm{F}_{\mathrm{br}}=1+1 \mathrm{~N}, \mathrm{~F}_{\mathrm{tr}}=20\right.$ $\mathrm{N})$ ensured continuous contact between the ulna and the roller over the full range of motion. In configuration $\mathrm{B}$, a roller was located anteriorly with respect to the ulna and induced flexion or extension of the forearm under a predominant flexor force $\left(\mathrm{F}_{\mathrm{hi}}+\right.$ $\mathrm{F}_{\mathrm{br}}=10+10 \mathrm{~N}, \mathrm{~F}_{\mathrm{tr}}=2 \mathrm{~N}$ ).

To map the envelope of joint laxity, a small constant varus or valgus moment $(0.5 \mathrm{Nm})$ was applied by a constant-force torsional spring. This spring was mounted on a lateral or medial extension affixed to the rotating roller and connected to the ulna. The resulting force vector therefore was perpendicular to the

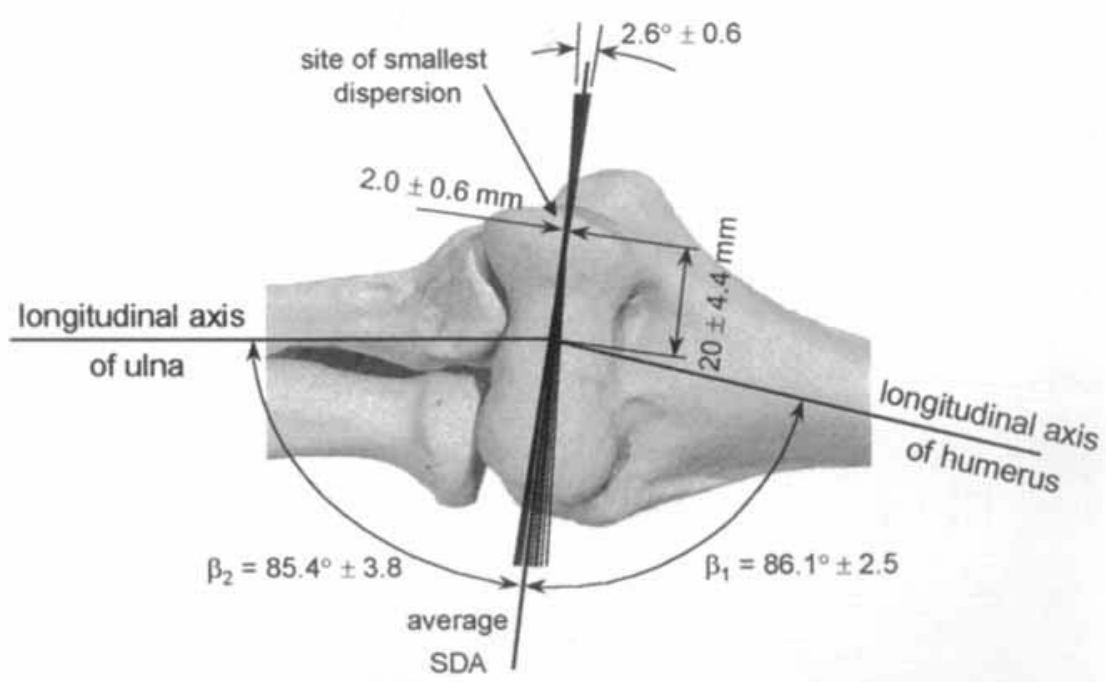

FIG. 4. Location of the calculated average screw displacement axis (SDA) with respect to the humerus and ulna in the frontal plane. $\beta_{1}$ denotes the angle betwcen the screw displacement axis and the longitudinal axis of the humerus. $\beta_{2}$ denotcs the angle between the screw displacement axis and the longitudinal axis of the ulna. The carrying angle is $\beta_{1}+\beta_{2}$. The excursion of the screw displacement axis $\left(2.6^{\circ}\right.$ ) is shown exaggerated by a factor of 2.0 to help visualize the site of the smallest dispersion of the axis. 


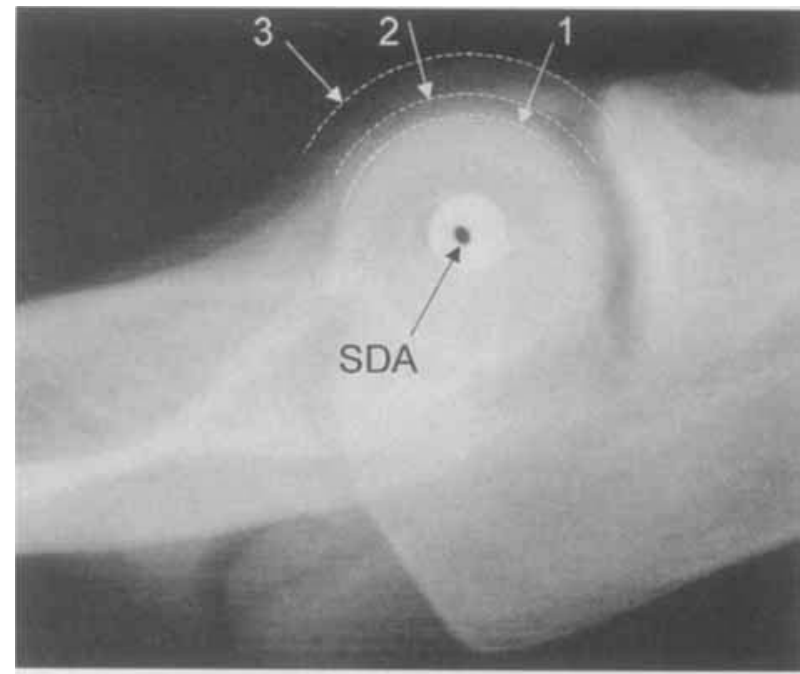

FIG. 5. Radiographic appearance of the elbow with an $x$-ray beam sited precisely along the average screw displacement axis (SDA). The rotation axis lics in the center of three concentric-appearing shadows formed by (1) the bottom of the trochlear sulcus, (2) the periphery of the capitellum, and (3) the medial facet of the trochlea.

ulna and remained perpendicular over the entire range of motion to a plane containing the humerus and the forearm.

The kinematics of rotation around the elbow joint were recorded with a direct-current electromagnetic tracking system (Flock of Birds, model 6DFOB; Ascension Technology, Burlington, VT, U.S.A.). This instrument traced the motion of a receiver coil triad with respect to a fixed transmitter operating at a sampling rate of $58 \mathrm{~Hz}$. The transmitter was rigidly connected to the humerus. The receiver was attached to the mid-diaphysis of the ulna. The receiver output completely described the threedimensional kinematics of the ulna with respect to the humerus, with translational and rotational accuracy of $0.5 \mathrm{~mm}$ and $0.1^{\circ}$, respectively (12). To avoid distortion of the motion-tracking recordings due to interference from nearby ferromagnetic objects $(4,12)$, the entire experimental setup was built from nonmagnetic materials (e.g., Plexiglas and fiberglass) and the direct-current motor was remote $(90 \mathrm{~cm})$ from the transmitter. Receiver recordings of flexion and then of extension of the elbow were obtained for roller configurations A and B. Each recording was repeated with the addition of a superimposed varus moment and then a valgus moment. Additional receiver recordings were collected to document trial-to-trial reproducibility, compatibility of recordings from intact or dissected specimens, and the influcnce of alternative motion protocols (roller configuration A compared with $\mathrm{B}$ and flexion compared with extension).

Elbow motion was completely described in terms of rigid-body screw displacement axes calculated for incremental rotations of $5^{\circ}$ with Beggs' (3) algorithm in combination with a customized procedure for smoothing source data (4). For each specimen, a bcst-fit screw displacement axis was calculated by averaging all screw displacement axes obtained over the entire range of motion. This average axis was used as a datum from which changes in orientation and translation of the screw displacement axcs were referenced.

Finally, the average screw displacement axis was physically marked in each specimen. A 4-mm holc was drilled with a customized drill guide into the trochlea along the axis. To avoid migration due to the oblique insertion angle, this was done with a four-flute end mill. A brass tube with an outer diameter of $4 \mathrm{~mm}$ and an inner diameter of $1.5 \mathrm{~mm}$ was inserted into the hole. Using fluoroscopy, we visualized the location of the average screw displacement axis with respect to the trochlea by sighting down the tube lumen. We then recorded the radiographic appearance of the distal humerus from the viewpoint of an x-ray beam oriented preciscly along the average screw displacement axis. Anteriorposterior radiographs were used to obtain the longitudinal axis of the humerus and ulna by fitting a line to the geometric center of the diaphyseal shaft. To enable further correlation between the screw displacement axis and anatomical structures, the location of the medial border of the trochlear facet and the lateral border of the capitellum were digitized. This experimental work, involving human cadaveric specimens, was approved by the institutional review board committee.

\section{RESULTS}

The pathway of the screw displacement axis for an elbow undergoing flexion over a range of motion from 10 to $130^{\circ}$ in roller configuration $\mathrm{A}$ is shown in Fig. 2. For visualization, the excursion of the axis is projected with scaling exaggeration onto intercept planes 200
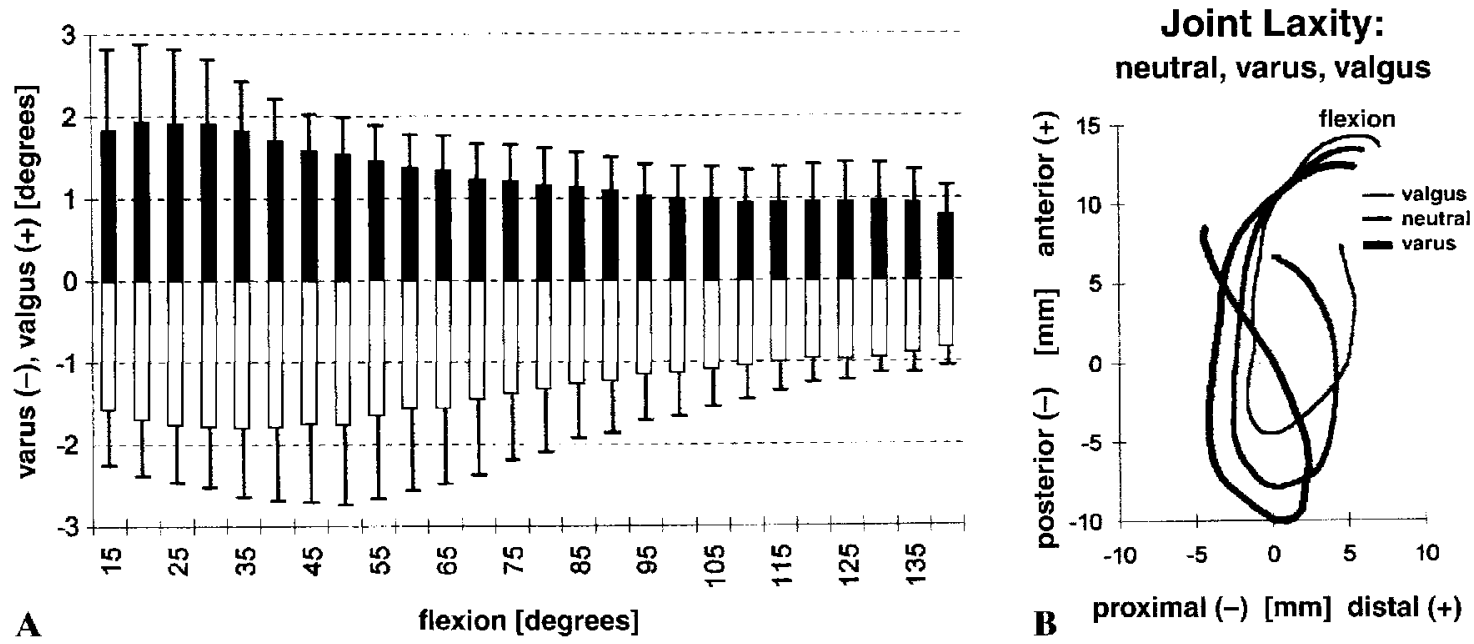

FIG. 6. A: Varus and valgus deviation from the normal motion path due to an applied varus or valgus moment of $0.5 \mathrm{Nm}$ shown at motion increments of $5^{\circ}$ over a range of motion of $15-140^{\circ}$ (average and standard deviation of seven specimens). B: Influence of varus and valgus moment on the normal pathway of the screw displacement axis. The pathway shown is the screw displacement axis intercept with a plane located $200 \mathrm{~mm}$ lateral of the trochleo-capitellar mid-sagittal plane. 
TABLE 1. Frustum parameters and waist location for neutral elbow flexion (roller configuration A) compared with flexion under applied varus or valgus moments of $0.5 \mathrm{Nm}$

\begin{tabular}{cccccc}
\hline $\begin{array}{c}\text { Flcxion } \\
\text { mode }\end{array}$ & $\mathrm{t}_{\mathrm{h}}(\mathrm{mm})$ & $\mathrm{t}_{\mathrm{f}}(\mathrm{mm})$ & $\left.\alpha_{\mathrm{h}}()\right)$ & $\alpha_{\mathrm{f}}\left({ }^{\circ}\right)$ & $\begin{array}{c}\text { Frustum waist } \\
\text { location }(\mathrm{mm})\end{array}$ \\
\hline Neutral & $1.4 \pm 0.3$ & $2.0 \pm 0.6$ & $5.7 \pm 2.2$ & $2.6 \pm 1.0$ & $19.9 \pm 4.4$ \\
Varus & $1.8 \pm 0.6$ & $2.0 \pm 0.7$ & $7.3 \pm 2.3$ & $4.2 \pm 1.7$ & $18.7 \pm 8.3$ \\
Valgus & $1.6 \pm 0.4$ & $2.1 \pm 0.8$ & $6.8 \pm 1.9$ & $2.6 \pm 0.7$ & $26.0 \pm 8.6$ \\
\hline
\end{tabular}

$t_{h}$ and $t_{f}=$ screw displaccment axis translations, and $\alpha_{h}$ and $\alpha_{\mathrm{f}}=$ frustrum vertcx angles in the horizonal and frontal planes, respectively.

${ }^{a}$ Frustum waist location is given in nillimeters medial of a trochleo-capitellar midsagitlal plane, obtained for a normalized trochleocapitellar width of $50 \mathrm{~mm}$.

$\mathrm{mm}$ medial and lateral from the geometric center of the trochlea.

The (axode) pathway of the screw displacement axis traces the surface of a double quasi-conic frustum of a nominally elliptical cross section (Fig. 3). To distinguish between translation and orientation changes of the screw displacement axis, the complex threedimensional excursion of the axis over the range of motion was differentiated into translations $t_{h}$ and $t_{f}$ in the horizontal and frontal planes, respectively, as determined by the dimension of the frustum waist, and the changes in orientation were depicted by the frustum vertex angles $\alpha_{\mathrm{h}}$ and $\alpha_{\mathrm{t}}$. These frustum parameters are reported for roller configuration $\mathrm{A}$. In the absence of varus/valgus moment over this range of motion, the screw displacement axis experienced excursions in the frontal plane (which includes the longitudinal axis of the fixed humerus) of $\mathrm{t}_{\mathrm{f}}=2.0 \mathrm{~mm}(\mathrm{SD}=0.62 \mathrm{~mm})$ and $\alpha_{f}=2.6^{\circ}\left(\mathrm{SD}=0.6^{\circ}\right)$. The excursions in the horizontal plane were $t_{h}=1.4 \mathrm{~mm}(\mathrm{SD}=0.32 \mathrm{~mm})$ and $\alpha_{h}=5.7^{\circ}$ $\left(\mathrm{SD}=2.19^{\circ}\right)$. The center of the frustum waist was located $20 \mathrm{~mm}(\mathrm{SD}=4.4 \mathrm{~mm})$ medial to a parasagit-

\section{Replicate SDA-Intercept Pathway flexion, extension}

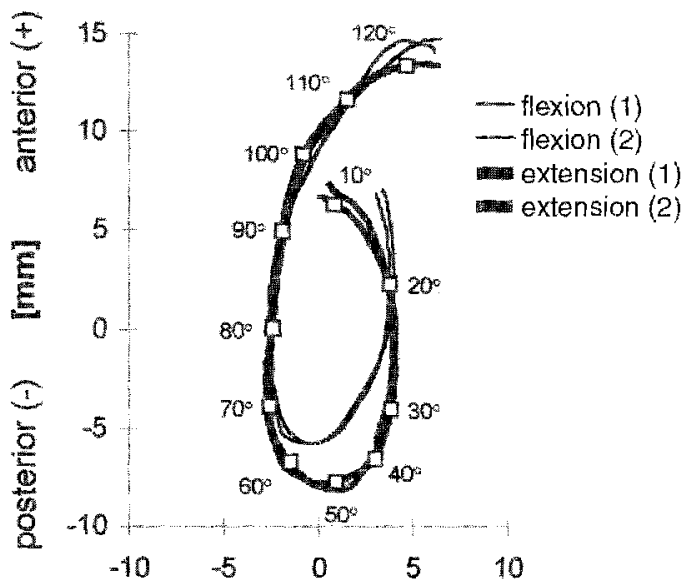

A proximal (-) Imm] distal (t) tal plane centered between the medial border of the trochlear facet and the lateral border of the capitellum. The location of the frustum waist was obtained by normalizing the trochleo-capitellar width to the series average of $50 \mathrm{~mm}$ and corresponds to a point within the medial facet of the trochlea.

The calculated average screw displacement axis penetrated the inferior anterior aspect of the medial epicondyle, the center of the trochlea, and the center of the projection of the capitellum onto a parasagittal plane. For the fully extended elbow viewed in the frontal plane, the average screw displacement axis formed an angle of $86.1^{\circ}\left(\mathrm{SD}=2.5^{\circ}\right)$ with the longitudinal axis of the humerus and an angle of $85.4^{\circ}$ ( $\mathrm{SD}=$ $3.8^{\circ}$ ) with the longitudinal axis of the ulna (Fig. 4). In a medial-lateral radiographic view (Fig. 5), with the $\mathrm{x}$ ray beam axis oriented precisely along the average screw displacement axis, the axis lies in the center of three concentric-appearing shadows. These shadows are formed by the bottom of the trochlear sulcus, the periphery of the capitellum, and the medial facet of the trochlea, respectively, as determined in a (sub-

\section{Roller Configuration A / B: flexion, extension}

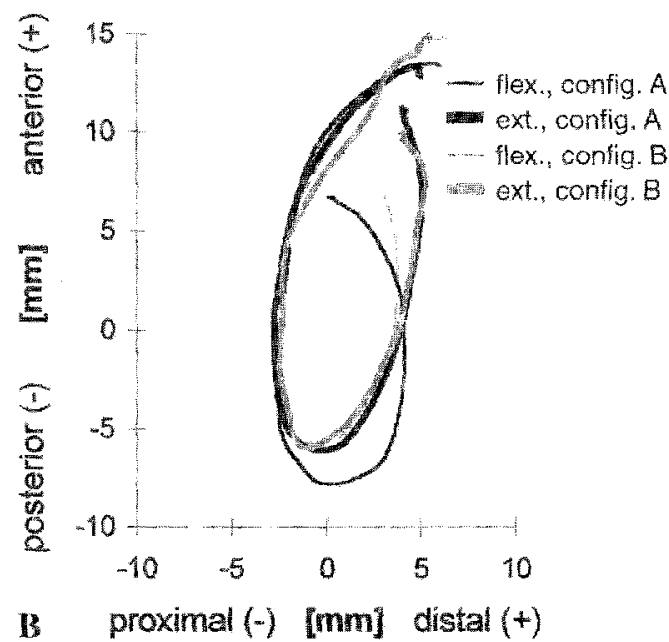

FIG. 7. A: Typical replicate screw displacement axis (SDA) pathways obtained from a single specimen. The direction of applied motion is designated as flexion or extension in roller configuration A. B: Typical screw displacement axis pathways in flexion and extension motion for the two roller configurations. 
TABLE 2. Sensitivity of the reported kinematic results to experimental parameters

\begin{tabular}{|c|c|c|c|c|c|c|c|c|c|c|c|}
\hline \multirow[b]{3}{*}{ Load } & \multirow[b]{3}{*}{ Specimen } & \multirow[b]{3}{*}{ Motion } & \multirow[b]{3}{*}{ Roller } & \multirow{2}{*}{\multicolumn{4}{|c|}{ Frustum parameters }} & \multicolumn{4}{|c|}{ Location of average SDA } \\
\hline & & & & & & & & \multirow{2}{*}{$\begin{array}{l}\text { Prox. (+) } \\
\text { Dist. (-) } \\
\text { (mm) }\end{array}$} & \multirow{2}{*}{$\begin{array}{l}\text { Ant. (+) } \\
\text { Post. (-) } \\
\text { (mm) }\end{array}$} & \multirow{2}{*}{$\begin{array}{l}\text { Int. rotation }(+) \\
\text { Ext. rotation }(-) \\
\left({ }^{\circ}\right)\end{array}$} & \multirow{2}{*}{$\begin{array}{c}\text { Eversion }(+) \\
\text { Inversion }(-) \\
\left(^{\circ}\right)\end{array}$} \\
\hline & & & & $t_{\mathrm{h} .}(\mathrm{mm})$ & $u_{1}(\mathrm{~mm})$ & $\alpha_{1}\left({ }^{\infty}\right)$ & $a_{f}\left({ }^{\circ}\right)$ & & & & \\
\hline Neutral & Dissceted & Flexion & $A$ & $1.3 \pm 0.35$ & $2.0 \pm 0.08$ & $6.7 \pm 0.17$ & $3.9 \pm 0.12$ & $0.0 \pm 0.08$ & $0.0 \pm 0.03$ & $0.0 \pm 0.10$ & $0.0 \pm 0.01$ \\
\hline Varus & Dissected & Flexion & A & 1.4 & 2.0 & 9.8 & 3.8 & -0.3 & -0.2 & 0.9 & -0.9 \\
\hline Valgus & Dissceted & Flexion & $A$ & 3.3 & 1.) & 4.7 & 3.5 & -0.2 & -0.6 & 0.7 & 0.4 \\
\hline Neutral & Not dissected & Flexion & A & 1.6 & 2.0 & 6.6 & 3.5 & 0.1 & -0.4 & 0.0 & -0.1 \\
\hline Neutral & Dissected & Extension & A & 1.3 & 2.0 & 5.8 & 3.0 & -0.1 & $-0) .7$ & -0.4 & 0.2 \\
\hline Neutral & Dissected & Flexion & B & 1.7 & 2.5 & 3.8 & 4.2 & 0.1 & 0.0 & -0.4 & 0.6 \\
\hline
\end{tabular}

$\iota_{t}$ and $t_{\mathrm{f}}=$ screw displacement axis translations, and $\alpha_{h}$ and $\alpha_{f}=$ frustrum verlex angles in the horizonal and frontal planes, respectively.

"The location of the average screw displacement axis (SDA), as it intersects the midsagital plane of the trochlea, is measured relative to the midsagittal intersection for recordings (row 1 ) of elbow flexion in roller configuration A for the dissected specimen.

sequent) lead-marker study (9). However, locating these landmarks sometimes proved problematic. In three of the seven specimens, the circular shadows of the trochlear sulcus and the capitellum ovcrlapped and could not be distinguished; in two of these three specimens, the medial facet of the trochlea was not detectable.

The deviation of the screw displacement axis from the normal (neutral) motion path (i.e., flexion motion in roller configuration $\mathrm{A}$ ) due to an applied varus or valgus moment of $0.5 \mathrm{Nm}$ was recorded for motion increments of $5^{\circ}$ over a range of motion of $15-140^{\circ}$ (Fig. 6A). The range of joint laxity toward varus and valgus moments was nominally symmetric with respect to the neutral motion path. Joint laxity was greatest within the initial $40^{\circ}$ of flexion (to 1.8 and $1.9^{\circ}$ of varus and valgus rotation, respectively) and decreased by roughly $50 \%$ for flexion angles exceeding $100^{\circ}$. The effect of the varus/valgus moment on the elbow rotation axis is alternatively depicted in Fig. $6 \mathrm{~B}$ in terms of the pathway of the screw displacement axis intercept with a plane located $200 \mathrm{~mm}$ lateral of the trochlear center. The corresponding values for $t_{b}, t_{f}$ $a_{h}$, and $a_{f}$ and the location of the frustum waist are listed in Table 1.

The screw displacement axis intercept with the 200$\mathrm{mm}$ lateral plane was further used to illustrate trialto-trial reproducibility (depicted by two sequential elbow flexions and extensions of one specimen, Fig. $7 \mathrm{~A}$ ) and the influence of the roller configuration (Fig. 7B). Quantitative assessment of the reproducibility of the reported kinematic results (location of the screw displacement axis and $t_{h}, t_{\mathrm{f}}, \alpha_{\mathrm{h}}$, and $\alpha_{\mathrm{f}}$ ), as well as their sensitivity to various experimental parameters, are summarized in Table 2 . These results were obtained from a single specimen, tested first before dissection, then when dissected, and then in various motion modes. The reported location of the average screw displacement axis was measured relative to the average of three repetitive recordings of elbow flexion in roller configuration $\mathrm{A}$ of the dissected specimen (Table 2 , row 1 , depicting trial-to-trial reproducibility).

\section{DISCUSSION}

Real-time electromagnetic motion tracking enabled thorough quantitative assessment of the complex kinematics of the elbow joint. The rigid-body motion of the ulna with respect to a coordinate system affixed to the humcrus was expressed in a unique and complete manner in terms of the screw displacement axis, generating results in a clinically conventional format that is applicable to arthroplasty or fixator application, or both.

The present findings are consistent with previous literature. Fischer's (9) original study, later quoted by Steindler (17), used tracings of the spatial motion of the tips of three needles affixed to the ulna to identify distinct rotation axes that intersected slightly medial of the trochlear center. Fischer described these axes as lying on a cone of irregular cross section with a vertex angle of $4-10^{\circ}$ and with a cone-tip excursion of $1-2$ $\mathrm{mm}$. Deland et al. (8) found the instant center of rotation to be within circles 3 and $1 \mathrm{~mm}$ in diameter on the lateral and medial sides of the elbow, respectively. They detcrmined that the average axis of rotation for five specimens formed an angle of $80 \pm 3^{\circ}$ with the longitudinal axis of the humerus, whereas Tanaka et al. (18) reported an angle of $84.3 \pm 1.7^{\circ}$ between an optimum axis of rotation and the longitudinal axis of the humerus. London (11) identificd a single rotation axis for the elbow that formed an angle of $82-86^{\circ}$ with the humerus. He reported that only for the last $5-10^{\circ}$ of the range of motion did the axis of rotation migrate proximally, a phenomenon that he attributed to a change from sliding to rolling joint motion accompanied by partial widening of the joint space. Although he noted the appearance of three concentric shadows on "true lateral" roentgenograms, the inconsistent visibility of these landmarks, especially of the medial facet of the trochlea, was not mentioned. 
O'Driscoll et al. (16) reported a maximum of $2.7 \pm$ $1.5^{\circ}$ varus/valgus joint laxity due to applied varus/valgus moments of $1.2 \mathrm{Nm}$. Tanaka et al. (18) observed that varus/valgus laxity was greatest at $20-30^{\circ}$ of flexion and that it decreased during further flexion. They reported an average total varus/valgus laxity to $7.6^{\circ}$ due to a moment caused by the weight of the forearm (estimated to be $2.2 \mathrm{Nm}$ ). To provide a point of reference for their findings, we additionally subjected one specimen to $0.4-\mathrm{Nm}$ incremental varus or valgus moments to $2 \mathrm{Nm}$. This resulted in an average total laxity of $13.3^{\circ}$ for the $2-\mathrm{Nm}$ load. Although tested for only one specimen, this suggests a higher varus/valgus laxity than that reported by Tanaka et al. This difference is most likely attributable to the 2-fold higher compressive force $\left(F_{b i}, F_{b r}\right.$, and $\left.F_{b r}\right)$ used by Tanaka ct al., since muscle forces provide dynamic stability to varus and valgus loads (16). Our applied varus/valgus moment of $0.5 \mathrm{Nm}$ is considerably smaller than the moment generated by the weight of the forearm itself $(2.0-2.4 \mathrm{Nm})(20)$ if clbow motion occurs in the horizontal plane.

The muscle-load configuration used in this study was chosen to simultaneously satisfy two requirements. It had to ensure continuous contact between the roller and the ulna and yet the total applied forces had to be kept minimal, to permit passive motion along a motion path constraincd primarily by the articular surfaces and ligamentous structures. Therefore, a minimal predominant flexor or extensor force of $20 \mathrm{~N}$ was necessary to achieve continuous roller-ulna contact over the entire range of motion. To avoid potentially confounding viscoelastic effects, the present study treated muscle forces $\left(\mathrm{F}_{\mathrm{bi}}, \mathrm{F}_{\mathrm{hr}}\right.$, and $\left.\mathrm{F}_{\mathrm{tr}}\right)$, forearm orientation (supination), and flexion rate $(32 \% \mathrm{sec})$ as well defined constants rather than as independent experimental variables. The minimally constrained application of passive motion enabled the elbow to trace a unique, neutral path. This particular path, however, was dependent on experimental parameters such as the muscle-load configuration, the applied varus/valgus moments, and the direction of elbow rotation. Given the vast array of possible muscle-load configurations during active elbow motion and the apparent envelope of joint laxity, conceivable in vivo kinematics can deviate considerably from the reported neutral motion path. Only one specimen was tested with both an intact and a dissected soft-tissue envelope, limiting the sensitivity summary in Table 2 to one specimen, albeit one tested for all experimental parameters.

Although the present results agree well with previous research (19), they constitute a substantial refinement of characterization detail. The screw displacement axis approach allows the location as well as the orientation of the pathway of the instant axis of elbow rotation to be tracked. The combined per- formance of the minimally constraining joint-motion applicator, the screw displacement axis algorithm, and the accuracy of the motion-tracking system was reflected in the good intra-specimen reproducibility of the frustum parameters, which was better than 0.4 $\mathrm{mm}$ and $0.2^{\circ}$ (Table 2, row 1 ).

The pathway of the screw displacement axis intercept proved to be a sensitive outcome parameter, adequate to qualitatively demonstrate differences in elbow kincmatics with respect to joint loading (neutral or varus/valgus moment, Fig. $6 \mathrm{~B}$ ), joint motion (flexion or extension, Fig. 7A) and load configuration (roller configuration A or B, Fig. 7B). However, the sensitivity trials revealed that none of the experimental parameters (flexion or extension, neutral or varus' valgus moment, dissected or not dissected, and roller configuration $\mathrm{A}$ or $\mathrm{B}$ ) caused the average axis to change more than $0.7 \mathrm{~mm}$ in position or $1^{\circ}$ in orientation. Thereforc, the average screw displacement axis proved to be efficient for closcly approximating the complex elbow kinematics in terms of a single fixed axis.

For purposes of total elbow arthroplasty, the present data rigorously describe the motion path and the amount of varus/valgus laxity in the natural elbow joint. Prosthesis design cannot be based solely on kinematic considerations; however, the present results suggest a need for accurate hinge placement for operative techniques that impose an artificial hinge to the elbow (6). Furthermore, the radiographic appearance as viewed from along the average screw displacement axis provides anatomic landmarks of clinical utility for optimal application of hinged elbow prostheses, hinged-joint distraction arthroplasty, or hinged external fixation, or all three $(5,7)$.

Acknowledgment: Financial assistance was provided by a grant from EBI Medical Systems, Parsippany, New Jersey.

\section{REFERENCES}

1. An KN, Hui FC, Morrey BF, Linscheid RL, Chao EY: Muscles across the elbow joint: a biomechanical analysis. I Biomech 14:659-669, 1981

2. An KN, Jacobsen MC, Berglund LJ, Chao EY: Application of a magnctic tracking device to kinesiologic studies. $J$ Biomech 21:613-620, 1988

3. Beggs JS: Kinematics. Berlin, Springer-Verlag, 1983

4. Botllang M, Marsh JL, Brown TD; Factors influencing accuracy of screw displacement axis detection with a D.C.-based electromagnetic motion tracking system. I Biomech Eng 120:431-435, 1998

5. Bottlang M, O'Rourke M, Steyers CM, Marsh JL, Brown TD: Qualitative validation of radiographic landmarks of the elbow rolation axis. Trans 22nd Am Soc Biomech, pp 521-522, 1998

6. Bottlang M, Madey SM, Steyers CM, Marsh JL, Brown TD: Hinged external elbow fixation: optimal axis alignment to minimize motion resistance. Trans Orthop Res Soc 24:494, 1999

7. Bottlang M, O'Rourke M, Steycrs CM, Marsh JL, Brown TD: Radiographic landmatks of the rotation axis of the humeroulnar articulation. Trans Orthop Res Soc 24:367, 1999

8. Deland IT. Garg A, Walker PS: Biomechanical basis for el- 
bow hinge-distractor design. Clin Orthop 215:303-312, 1987

9. Fischer O: Handbuch der Anatomie und Mechanik der Gelenke, unter Berücksichtigung der Bewegenden Muskeln, vol 2, pp 299-308. Jena, Germany, Verlag Gustav Fischer, 1911

10. King GJ, Itoi E, Risung F, Niebur GI, Morrey BF, An KN: Kinematics and stability of the Norway elbow: a cadaveric study. Acta Orthop Scand 64:657-663, 1993

11. London JT: Kinematics of the elbow. J Bone Joint Surg [Am] 63:529-535, 1981

12. Milne AD, Chess DG, Johnson JA, King GJ: Accuracy of an electromagnetic tracking device: a study of the optimal operating range and metal interference. $J$ Biomech 29:791-793, 1996

13. Morrey BF, Chao EYS: Passive motion of the clbow joint. I Bone Joint Surg [Am] 58:501-508, 1976

14. Morrey BF: Distraction arthroplasty: clinical applications. Clin Orthop 293:46-54, 1993
15. Murray WM, Delp SL, Buchanan TS: Variation of muscle moment arms with clbow and forearm position. $J$ Biomech 28:513-525, 1995

16. O'Driscoll SW, An K-N, Korinek S, Morrey BF: Kinematics of semi-constrained total elbow arthroplasty. $J$ Bone Joint Surg [Br] 74:297-299, 1992

17. Steindler A: Kinesiology of the Human Body under Normal and Pathological Conditions, p 490. Springfield, Illinois, Thomas, 1955

18. Tanaka S, An KN, Morrey BF: Kinematics and laxity of ulnohumeral joint under varus-valgus stress. $J$ Musculoskel Res 2:45-54, 1998

19. Werner FW, An KN: Biomechanics of the elbow and forearm. IIand Clin 10:357-373, 1994

20. Winter DA: Biomechanics of Human Motion. New York, Wilcy, 1979 